\title{
DEFENCE REACTION IN DENTAL PULP AFTER PULP CAPPING AND PARTIAL PULPECTOMY IN DOGS
}

\author{
Z. PAVLICA ${ }^{1 *}$, Polona JUNTES ${ }^{2}$ and M. POGAČNIK ${ }^{2}$ \\ ${ }^{1}$ Small Animal Clinic and ${ }^{2}$ Institute of Pathology, Forensic and Administrative Veterinary \\ Medicine, Veterinary Faculty, University of Ljubljana, Gerbičeva 60, 1000 Ljubljana, \\ Slovenia
}

(Received April 21, 1999; accepted October 20, 1999)

\begin{abstract}
The dental pulp was capped indirectly or directly, or partial vital pulpectomy was performed on the 12 functionally most important teeth of 24 beagle dogs. For pulp capping, calcium hydroxide was used, followed by zinc phosphate as a lining, and the preparation was restored with amalgam or composite material. Histological sections were prepared and examined for degree and type of pulp inflammation (hyperaemia, pulpitis, necrosis or gangrene). Degenerative changes of dental pulp (vacuolation, calcification, amyloid or hyaline changes) were also determined. For indirect pulp capping the width of predentine in crown and root dental pulp was measured, and for direct pulp capping and partial pulpectomy (vital pulpotomy) the width and quality of the dentinal bridge were graded. The radical method of partial vital pulpectomy of the coronal part of the dental pulp in dogs produced better quality and continuity of the dentinal bridge than the less radical method of direct pulp capping. This was particularly obvious in small single root teeth such as incisors.
\end{abstract} tomy, dog

Key words: Dental pulp, defence reaction, pulp capping, partial pulpec-

The dental pulp has several functions including formative, nutritive and se nsory roles, defence-inflammatory reaction, and production of secondary dentine as recorded earlier by several authors (Mjor and Karlsen, 1970; Wenneberg et al., 1982; Ten Cate, 1985; Ivanović and Santini, 1989; Sicher and Bhaskar, 1990).

The reticuloendothelial barrier of the dental pulp is composed of reticular binding tissue in the cell-rich and cell-deficient layers and around blood vessels in the middle of the dental pulp, plus phagocytic capillary endothelium and macr ophages. According to Vrbošek (1981) and Heyeraas (1985), the main function of the reticuloendothelial barrier is its phagocytic activity. It is important for the binding of antigens, binding of macrophages to cells producing antigens, distin ction of normal and damaged tissue, and dete ction of alien materials.

*E-mail: PavlicZl@mail.vf.uni-lj.si; Fax: +386 (0) 61333708 
The dental pulp is subject to many injurious influences: mechanical trauma is the most frequent, such as attrition resulting from excessive wear of the teeth of working and sport dogs. Thermal trauma caused by overheating of the tooth during cavity preparation or finishing of restorations also causes pulp injury, as does chem ical injury resulting from inappropriate use of filling mat erials and medications.

Bacterial infection is a very important cause of damage. Most often bacteria attack the dental pulp centripetally through carious lesions or traumatic pulp exp osure in dogs and through periodontal communication. Bacteria can also enter the dental pulp by the haematogenous route, particularly when the resistance of the host organism is compromised.

Clinically the first signs of dental pulp inflammation are hyperaemia, fo 1lowed by painful symptomatic pulpitis that may develop into a painless but irr eversible condition. This leads to necrosis of the dental pulp. If the inflammation is rapidly controlled, the condition of the pulp tissue can return to normal. However, necrosis and gangrene occurring during the irreversible phase spread into periap ical tissues as described previously (Naidorf, 1972; Tronstad and Mjor, 1972; Tronstad, 1974; Tonder and Naess, 1979; Cvek et al., 1982; Tonder and Kvinnsland, 1983; Schroder, 1985).

Experimental infection of the dental pulp with bacteria is followed by local immune reaction, which can be cellular and humoral. The tissue is filled with ne utrophils, plasma cells, T lymphocytes, B lymphocytes, macrophages, and mast cells. Initially neutrophils and phagocytes are found. B lymphocytes release lymphokines that exert cytotoxic effects on fibroblasts and activate osteoclasts. Plasma cells produce immunoglobulins, while mast cells release histamine and other active substances. The effect of all these mediators is more destructive than protective.

Calcium hydroxide can be thought of as a direct bandage for damaged tissue. Its $\mathrm{pH}$ value of 12.5 produces superficial necrosis in the pulp tissue, followed by formation of a compact tissue barrier. Yamamura (1985) described that the initial $r$ eaction of pulp to calcium hydroxide occurs within one hour, and is seen as superficial coagulative necrosis at the edge of the vital pulp tissue. Heide and Mjor (1983) and Matsuzaki et al. (1990) described some experimentally provoked pulp reactions. They found that calcium carbonate, originating from the reaction of tissue $\mathrm{CO}_{2}$ and blood, obstructs the diffusion of hydroxyl ions and blocks the necrotic effect, pr otecting the dental pulp from further damage. The vessels in the pulp tissue are hype $r-$ aemic. Inflammatory cells enter the damaged tissue $6 \mathrm{~h}$ to several days later as described by Barker and Lockett (1971) and Cullum and Kline (1985). Granulomatous tissue with proliferation of fibroblasts and angioblasts (especially in the proximity of necrosis), or sterile abscess surrounded by macrophages can be observed. On the fourth day, new collagen appears beneath the necrotic layer, and after approximately seven days cellular inclusions are observed. This proliferative phase includes binding tissue that promotes formation of mature dentine. Odontoblasts are formed from pulp 
fibroblasts or reserve cells, and tertiary, canalised dentine is observed during the sixth week. Such a pulp response has been described by Mjor (1985), Ivanović and Santini (1989), and Matsuzaki et al. (1990).

The aim of the present study was to follow the dentinal pulp response of the most active teeth after indirect and direct pulp capping and after vital partial pu 1pectomy, to establish which method is the most successful and potentially more useful in the clinical rehabilitation of canine teeth.

\section{Materials and methods}

The dogs were tranquillised with $0.01 \mathrm{mg} / \mathrm{kg} \mathrm{b}$. w. acetylpromazine followed by a short-acting barbiturate (thiopental). After intubation, general anaesth esia was induced with inhalation anaesthetics.

All the teeth were first disinfected with $0.2 \%$ chlorhexidine. The tooth used in the experiment, both teeth next to it and the gingiva were also disinfected before each operation with povidone iodine.

For preparation of the enamel, diamond burs of round and pear shape were used $(0.9,0.12,0.14$, and 0.16 ISO standards). In dentine and in the crown part of the pulp chamber, carbide burs of round and inverted cone shape (0.12 and 0.14 ISO standards) were used. All the drilling instruments used in the experiment were sterilised in autoclave. During the experiments the burs were kept in Petri dishes containing formalin pills.

Indirect dental pulp capping (IPC) of upper right canine, upper fourth right premolar, upper left canine, lower left canine and lower first left molar was pe rformed in 24 beagle dogs of 2 years of age. On all teeth, a class V cavity was pr epared. The preparation was deep enough, so that only a thin layer of intact dentine was left over the pulp chamber as indicated by pink coloration of the dentine. A $0.5 \mathrm{~mm}$ thick calcium hydroxide paste was placed and covered with zinc phosphate cement, and the preparations were restored with amalgam (premolar and molar teeth) or composite materials (canine and incisor teeth) (Fig. 1).

On the lower left third incisors, lower right third incisors, upper left fourth premolars and lower right first molars, the dental pulp was exposed after prepar ation of a class $\mathrm{V}$ cavity. Bleeding was stopped by gently placing a sterile cotton pellet on the dental pulp for $3 \mathrm{~min}$. After bleeding had been controlled and fo 1lowing drying of the cavity and exposed dental pulp, a $1 \mathrm{~mm}$ thick calcium hydroxide paste was placed and covered with zinc phosphate cement. This was fo 1lowed by restoration with amalgam or composite (Fig. 2).

On the upper right third incisors, upper left third incisors and lower right canines, a class V cavity was prepared and the dental pulp was exposed. The same sterile bur was used to create a $2-3 \mathrm{~mm}$ partial pulpectomy. After washing the 
cavity with sterile physiologic saline solution and drying with a sterile cotton pe 1let, haemostasis was achieved by placing a sterile cotton pellet soaked in $30 \% \mathrm{~h} \mathrm{y}$ drogen peroxide for $1 \mathrm{~min}$, followed by repeated drying. The partial pulpectomy wound was treated with calcium hydroxide paste and covered with zinc phosphate cement. This was followed by standard restoration with composite (Fig. 3).

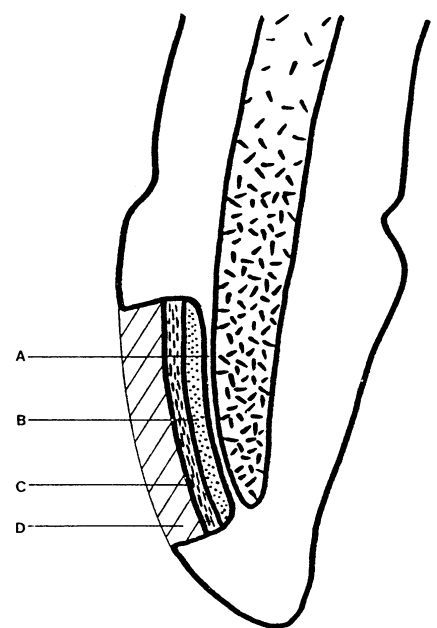

1

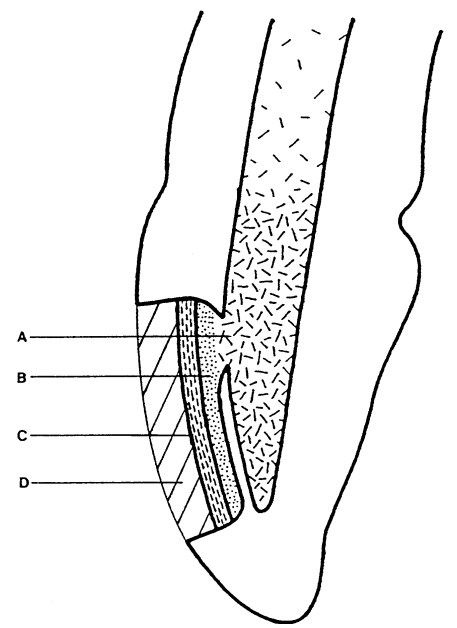

Fig. 1. Indirect pulp capping (schematic presentation). A: thin layer of intact dentine, B: calcium hydroxide paste, C: zinc phosphate cement, D: composite restoration Fig. 2. Direct pulp capping (schematic presentation). A: Exposed dental pulp, $\mathrm{B}$ : calcium hydroxide paste, C: zinc phosphate cement, D: composite restoration

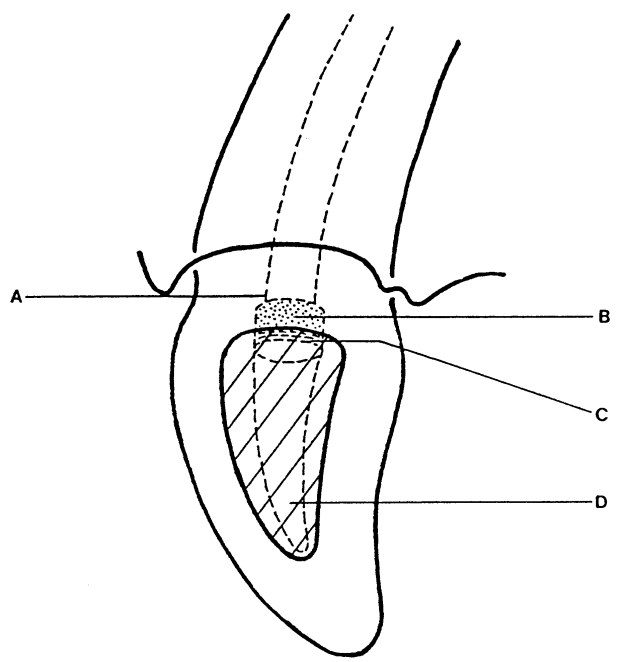

Fig. 3. Vital partial pulpectomy (schematic presentation). A: Partial pulpectomy wound, $\mathrm{B}$ : calcium hydroxide paste, $\mathrm{C}$ : zinc phosphate cement, D: composite restoration 
On the sixtieth day the test teeth were extracted under anaesthesia. After 72hour fixation in $10 \%$ neutral formalin, the teeth were demineralized in $88 \%$ formic acid and $20 \%$ sodium citrate solution. Paraffin blocks of the decalcified teeth were oriented to permit bucco-lingual sectioning of the teeth. Eight microns thick para ffin sections were made and stained with haematox ylin and eosin.

Statistical evaluation of the results was performed by the use of the SPSS (Statistical Package for the Social Sciences) program.

\section{Results}

Indirect pulp capping was performed on 55 teeth. Of them, 53 teeth were examined, because two teeth were damaged during extraction. A total of 277 histologic sections were exa mined.

In teeth treated by indirect pulp capping, calcium hydroxide stimulation of the dental pulp had formed a thick predentine layer. The thinner the dentine, the thicker was the layer of predentine beneath the prep aration (Table 1).

On 44 teeth the dental pulp was capped directly. Six teeth were damaged during extraction, and 233 histologic sections were examined. Chronic inflamm ation was seen in $30 \%$ of the upper left fourth premolars, $37 \%$ of the lower right first molars and $11 \%$ of the lower third left incisors. In two third incisors, necrosis with autolysis of dental pulp was found.

Table 1

Results of indirect pulp capping of the dental pulp in dogs (measurements are given in $\mu \mathrm{m}$ )

\begin{tabular}{lrrrrr}
\hline \multicolumn{1}{c}{ Teeth } & \multicolumn{1}{c}{ A } & B & C & D & E \\
\hline Upper right canines & 456.4 & $28.8 \pm 1.2$ & $10.5 \pm 0.3$ & $7.9 \pm 0.3$ & $6.0 \pm 0.3$ \\
Upper right fourth premolars & 1260.1 & $11.9 \pm 0.5$ & $5.7 \pm 0.1$ & $6.7 \pm 0.1$ & $4.4 \pm 0.1$ \\
Upper left canines & 785.6 & $13.3 \pm 0.4$ & $8.6 \pm 0.2$ & $6.3 \pm 0.2$ & $4.0 \pm 0.2$ \\
Lower left canines & 609.9 & $21.8 \pm 1.4$ & $9.7 \pm 0.3$ & $9.1 \pm 0.2$ & $5.8 \pm 0.2$ \\
Lower left first molars & 1341.9 & $9.6 \pm 0.6$ & $5.7 \pm 0.2$ & $4.8 \pm 0.1$ & $3.9 \pm 0.1$ \\
\hline
\end{tabular}

A: dentine on the floor of the cavity; B: predentine on the floor of the cavity; C: predentine on the opposite side of the cavity; D: predentine $5 \mathrm{~mm}$ apically on the floor of the cavity; E: predentine on the opposite side of the root canal

Vital amputation (partial pulpectomy) was performed on 33 teeth. One tooth broke during extraction. A total of 137 histologic sections were exa mined.

An incomplete dentinal bridge was found more frequently over the dental pulp in direct pulp-capped specimens compared with vital partial pu lpectomy.

Compared with direct pulp capping, inflammation was seen only on upper third left incisors treated by vital amputation of the coronal pulp (Table 2). 
Table 2

Comparative dentinogenic effects of calcium hydroxide in direct pulp capping (DPC) and vital amputation (VA) of the dental pulp in dogs (measurements are given in $\mu \mathrm{m}$ )

\begin{tabular}{lrccr}
\hline \multicolumn{1}{c}{ Teeth } & \multicolumn{1}{c}{ A } & B & C & D \\
\hline Upper left fourth premolars - DPC & $42.3 \pm 5.4$ & $22.8 \pm 2.90$ & $29.3 \pm 4.0$ & 30 \\
Lower left third incisors - DPC & $53.6 \pm 5.9$ & $82.3 \pm 10.5$ & $36.9 \pm 3.1$ & 11 \\
Lower right third incisors - DPC & $87.7 \pm 8.4$ & $64.0 \pm 6.9$ & $28.8 \pm 5.6$ & 0 \\
Lower right first molars - DPC & $78.5 \pm 8.0$ & $76.2 \pm 8.0$ & 0 & 37 \\
Upper right third incisors - VA & $182.2 \pm 9.4$ & $169.0 \pm 9.4$ & $66.0 \pm 8.0$ & 0 \\
Upper left third incisors - VA & $95.2 \pm 7.6$ & $80.9 \pm 7.9$ & $54.2 \pm 3.6$ & 11 \\
Lower right canines - VA & $147.4 \pm 9.6$ & $111.5 \pm 9.3$ & $65.5 \pm 12.2$ & 0 \\
\hline
\end{tabular}

A: granulomatous barrier (in $\mu \mathrm{m}$ ); B: incomplete dentinal barrier (in $\mu \mathrm{m}$ ); C: continuous barrier with well-organised tertiary dentine (in $\mu \mathrm{m}$ ); D: inflammation of the dental pulp (in \%)

\section{Discussion}

In teeth treated by indirect pulp capping, calcium hydroxide stimulation of the dental pulp had formed a thick predentine layer. The thinner the dentine, the thicker was the layer of predentine beneath the preparation. In the newly formed predentine the formation and mineralization began at the layer closest to the dentine-predentine border. On the pulp capping side the mineralization of predentine occurred at the normal distance from odontoblasts. The mineralization of predentine was greater than on other sides as a result of the calcium hydroxide effect. Strong mineralization of an old predentine was seen only on the pulp capping side (Fig. 4).

In the upper right fourth premolars, inflammation was seen only at the pulp capping site and had no suppressive dentinogenic effect, so that the predentine layer beneath the cavity was thicker. In these teeth, less severe inflammation pe rmitted the formation of predentine.

On the lower first left molars, generalized inflammation was present and predentine formation did not occur (Fig. 5). In these teeth the inflammation was probably the consequence of overheating during preparation of the cavity. At the pulp capping site in one tooth from this group, amorphous, reddish, round form ations of amyloid of different sizes, surrounded by inflammatory cells, were found under the odontoblast layer and around vessels in the connective tissue (Fig. 6). These formations, which appeared to be resistant to demineralizing processes, could represent hyaline material, which is also formed by chronic irritation or chronic inflammation and is the result of antigen-antibody reactions.

The dogs were not treated with antibiotics to protect the dental pulp from i nfection, as the aim was to determine the defence capability of the dental pulp in dogs. Absence of antibiotic treatment may explain the high incidence of severe, chronic inflammation in the teeth treated by $\mathrm{d}$ irect pulp capping. 


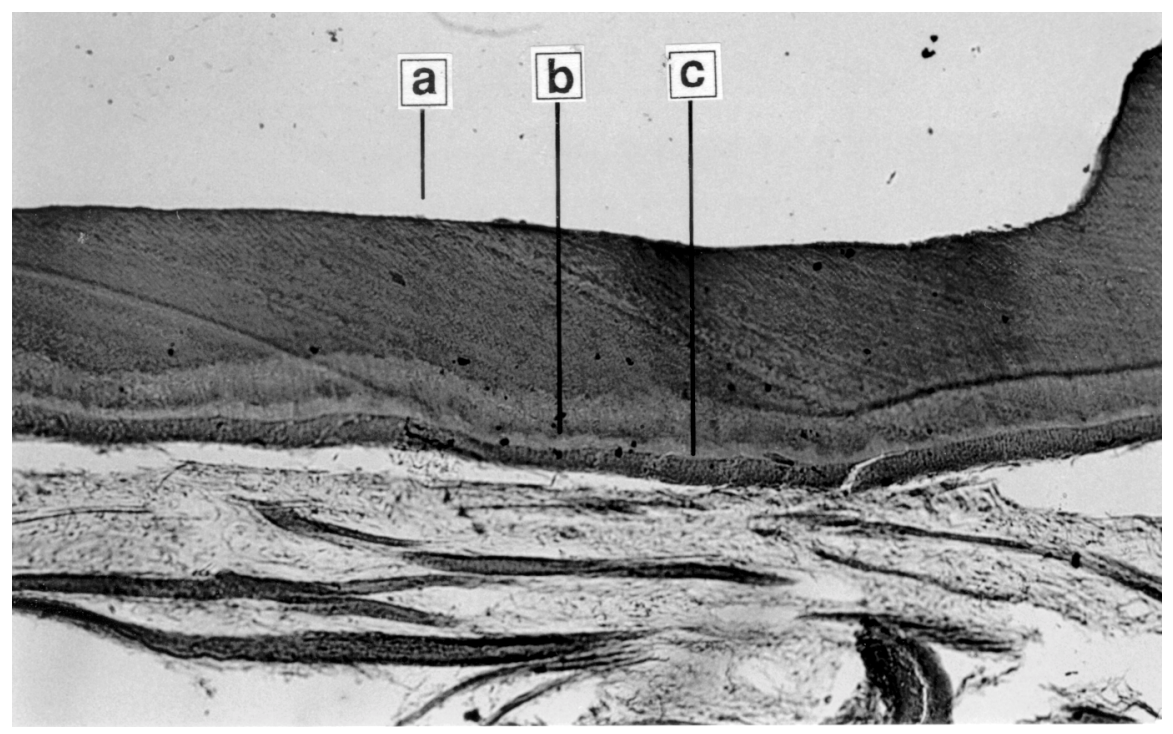

Fig. 4. Left upper canine 60 days after the procedure (original magnification: $\times 100$ ); a: prepared cavity; b: well-mineralized old predentine; c: extended thickness of predentine beneath the cavity

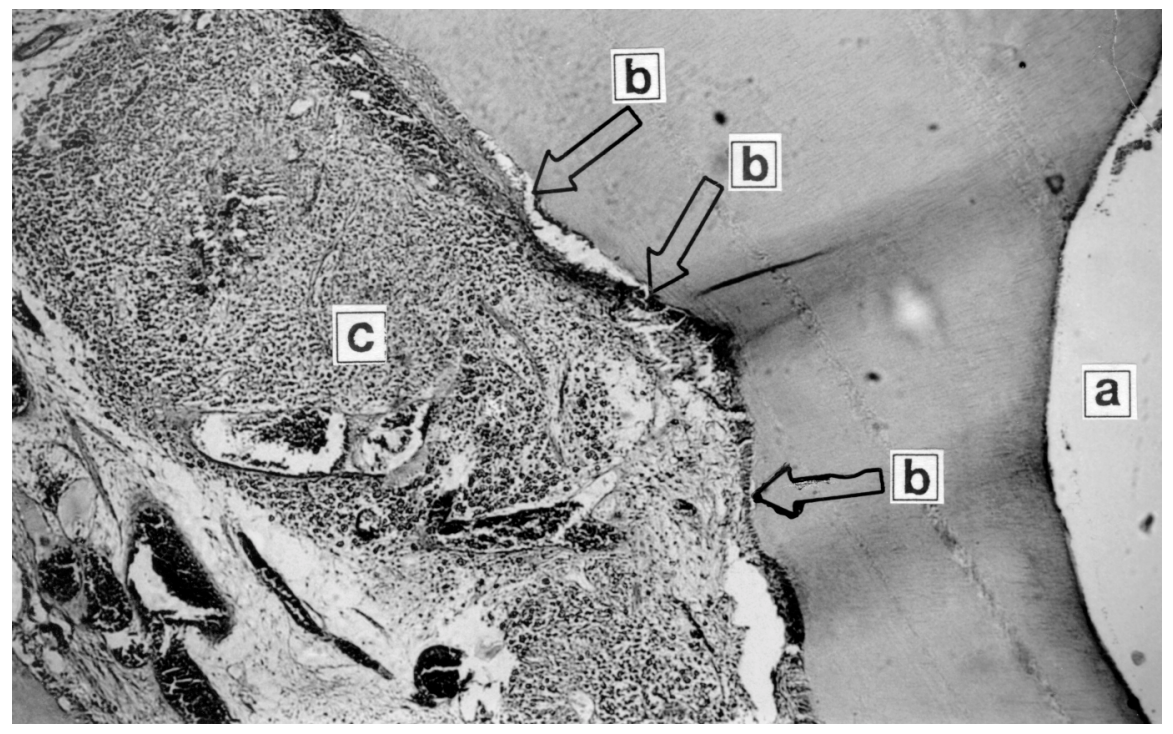

Fig. 5. Lower left first molar 60 days after the procedure (original magnification: $\times 60$ ); a: prepared cavity, b: no dentinogenic effect, c: generalized inflammation

The chronic granulomatous reaction persisted longer than with vital partial pulpectomy, suggesting that the tissues were unable to effectively remove necrotic products. It is also possible that the activity of calcium hydroxide is not restricted 
to local effects. Particles of calcium hydroxide may be washed from the initial site to elsewhere in the coronal pulp and coronal third of the root canal where, like fo reign material, they become surrounded by inflammatory cells (Fig. 7). The ce ntripetal movement of calcium hydroxide from the place of application can be e xplained by the abundant blood supply of the dental pulp in young mature dogs.

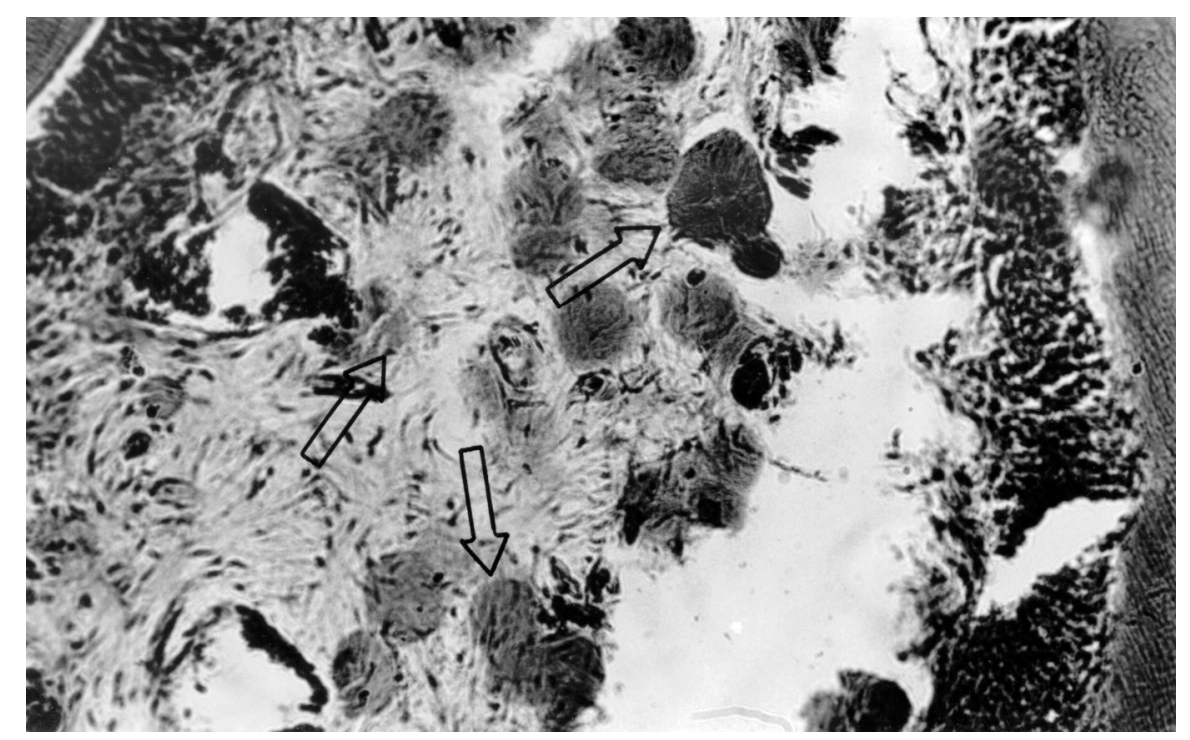

Fig. 6. Lower left first molar 60 days after the procedure (original magnification: $\times 200$ ). Note amyloid bodies near vessels and under the odontoblast layer (arrows)

An incomplete dentinal bridge was found more frequently over the dental pulp in direct pulp-capped specimens compared with vital partial pulpectomy (Fig. 8). This may be due to the presence of necrotic products that caused a granulomatous response resulting in a barrier composed of tertiary dentine resembling osteode ntine.

Compared with direct pulp capping, inflammation was seen only on upper third left incisors treated by partial pulpectomy of the coronal pulp. Decreased i nflammation may have resulted from the use of hydrogen peroxide as an antiseptic during the partial pulpectomy procedure. The presence of inflammatory cells e nclosing crystals of calcium hydroxide in crown and root canal pulp can be attri buted to chemical irritation caused by calcium hydroxide. As for findings with $\mathrm{d}$ irect capping, calcium hydroxide may have been distributed via the lymphatic or interstitial fluid, or may have been carried via blood vessels to the periapex and b eyond (Fig. 7). 
On the left third incisors, local inflammation of the coronal third of root $\mathrm{c}$ anal dental pulp was observed. However, a hard continuous barrier with wellformed tertiary dentine with dentinal tubules was seen (Fig. 9).

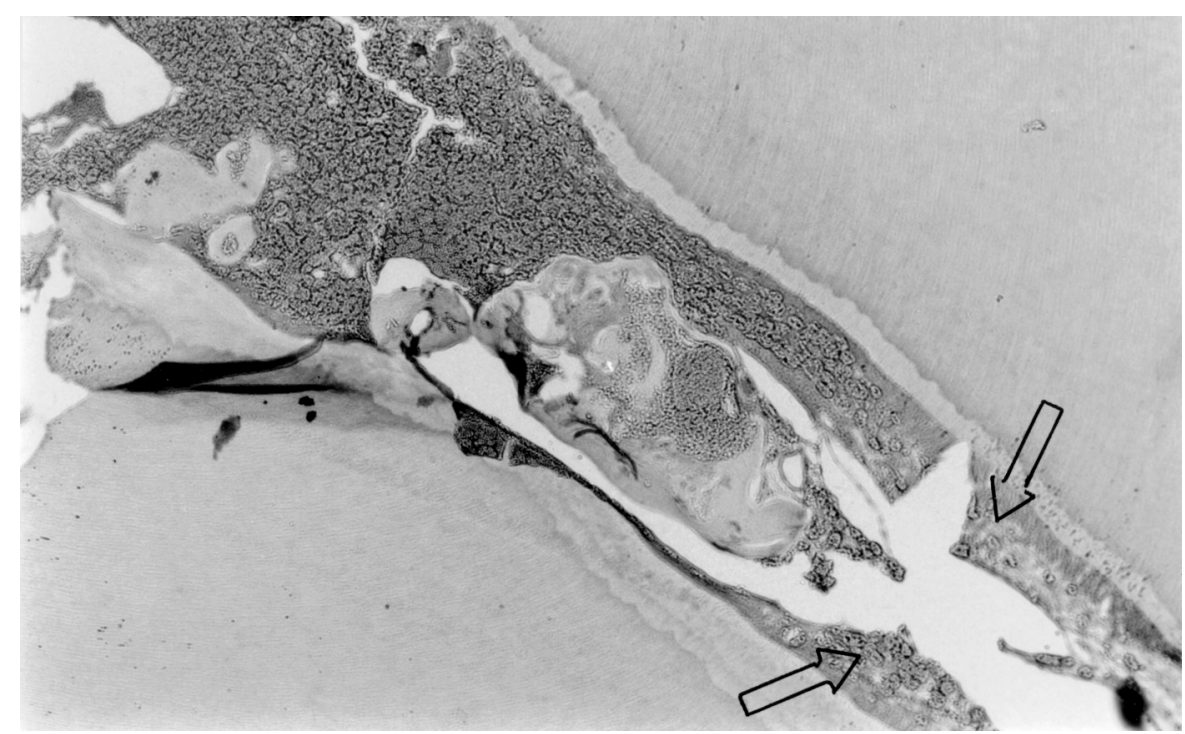

Fig. 7. Upper left third incisor 60 days after the procedure (original magnification: $\times 100$ ); calcium hydroxide has moved via lymph, interstitial fluid or blood vessels

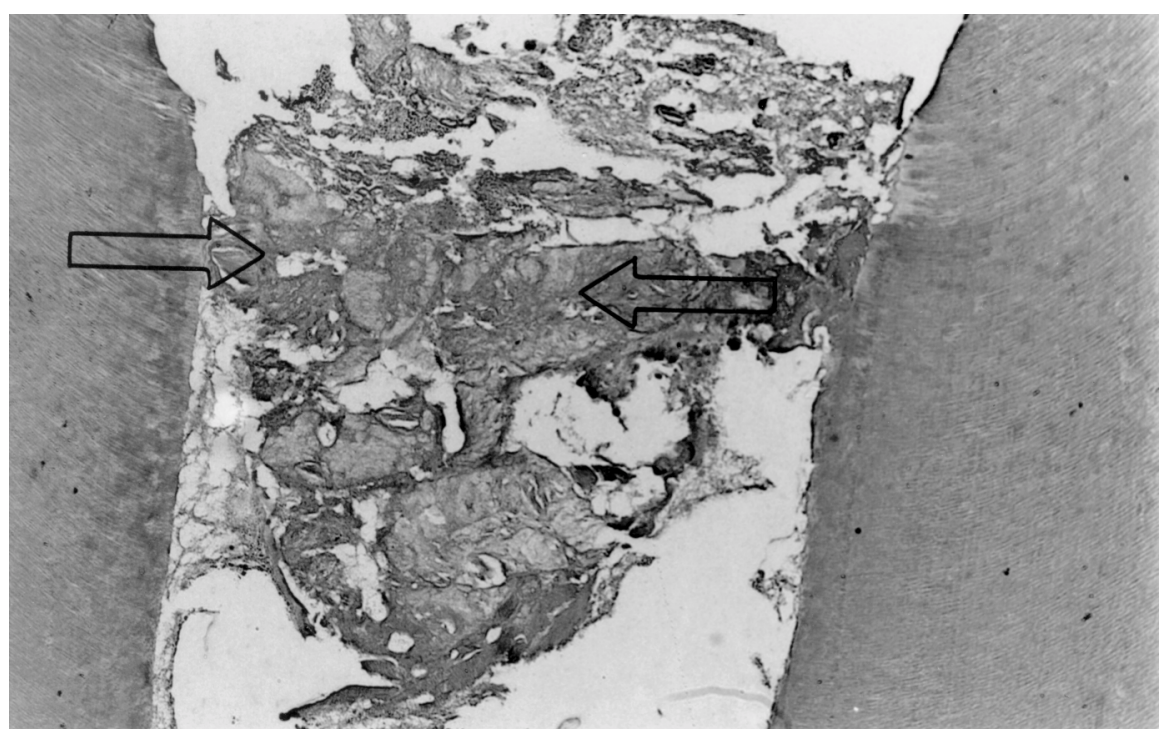

Fig. 8. Lower left third incisor 60 days after the procedure (original magnification: $\times 200$ ); incomplete barrier of tertiary dentine (arching dentinal tubules) 
Formation of tertiary dentine with dentinal tubules in a continuous barrier anywhere in the amputation area results from transformation of other pulp cells (fibroblasts, nondifferentiated mesenchymal cells) to odontoblasts, as described earlier by several authors (Mjor and Karlsen, 1970; Wenneberg et al., 1982; Mjor, 1985; Ten Cate, 1985; Matsuzaki et al., 1990). They build the organic matrix that is later mineralized into reparative dentine. In the present experiments the tertiary dentine was always formed initially laterally in a continuous barrier. The tertiary dentine differed from normal dentine in having fewer tubules, which were strongly curved (Fig. 10).

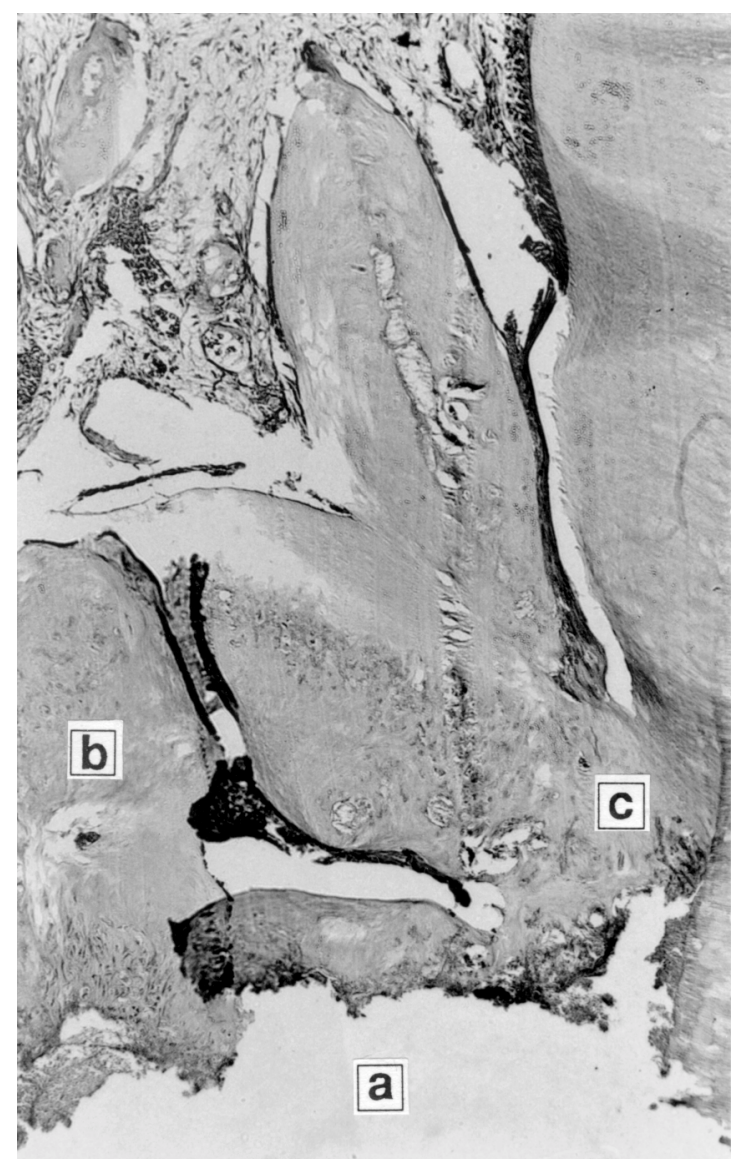

Fig. 9. Upper right third incisor 60 days after the procedure (original magnification: $\times 100$ );

(a) partial pulpectomy wound, continuous barrier of tertiary dentine (b) with and (c) without tubules 


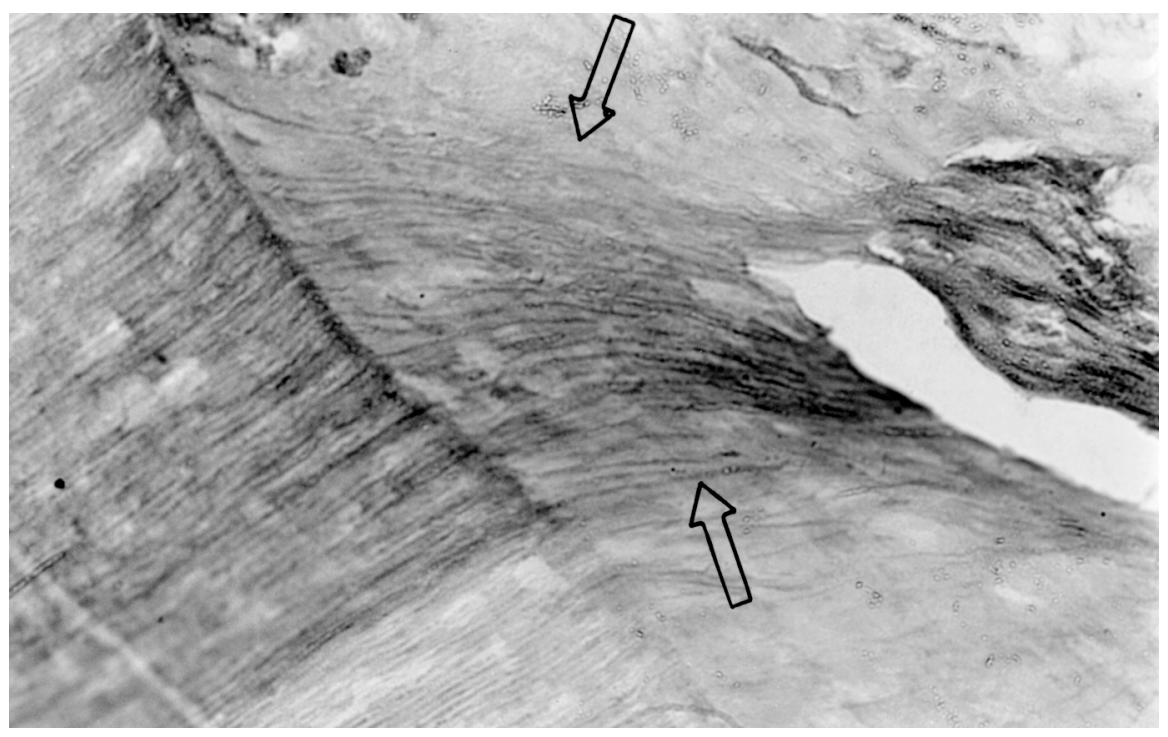

Fig. 10. Upper right third incisor 60 days after the procedure (original magnification: $\times 400$ ); well-organised tertiary dentine in a continuous barrier

\section{Conclusions}

In teeth with some dentine remaining on the floor of the preparation, $\mathrm{d} i$ rectly beneath it, the width of predentine was greater in comparison with prede $n$ tine of normal width on the opposite side. The inflammation of dental pulp, which was found only in the lower left first molars, resulted in less new predentine. The amorphous-round amyloid bodies found around vessels and under the odontoblastic layer probably resulted from local defence mechanisms. Comparison of the $\mathrm{r}$ esults between direct pulp capping and partial pulpectomy of the dental crown showed that the more radical method (partial pulpectomy) caused less inflamm ation and a thicker cover from well-formed, canalised tertiary dentine. Due to met abolic activity of the canine dental pulp and numerous vessels, the crystals of ca 1cium hydroxide were found apical to the capping site, where they were enclosed by inflammatory cells. On the basis of these results, the radical method of vital amputation-partial pulpectomy is recommended as the most suitable approach for retaining the vitality of teeth with directly exposed pulp. 


\section{References}

Barker, C. W. and Lockett, B. C. (1971): An unusual response of dog pulp to calcium hydroxide. Oral Surg. 32, 785-799.

Cullum, D. R. and Kline, L. W. (1985): Pulp response after calcitonin treatment of direct exposures in the dog. Oral Surg. Oral Pathol. 60, 218-223.

Cvek, M., Cleaton-Jones, P. E., Austin, J. C. and Andreasen, J. O. (1982): Pulp reactions to exposure after experimental crown fractures or grinding in adult monkeys. J. Endod. 8, 391-397.

Heide, S. and Mjor, I. A. (1983): Pulp reactions to experimental exposures in young permanent monkey teeth. J. Endod. 16, 11-19.

Heyeraas, K. J. (1985): Pulpal microvascular and tissue pressure. J. Dent. Res. 64 (Special Issue), $585-589$.

Ivanović, V. and Santini, A. (1989): Rate of formation of tertiary dentin in dogs' teeth in response to lining materials. Oral Surg. 67, 684-688.

Matsuzaki, K., Fuji, H. and Machida, Y. (1990): Experimental study of pulpotomy with calcium hydroxide - iodoform paste in dogs immature permanent teeth. Bull. Tokyo Dent. Coll. 31, 9-15.

Mjor, I. A. (1985): Dentin-predentin complex and its permeability. Pathology and treatment overview. J. Dent. Res. 64 (Special Issue), 621-627.

Mjor, I. A. and Karlsen, K. (1970): The interface between dentine and irregular secondary dentine. Acta Odontol. Scand. 28, 363-376.

Naidorf, I. J. (1972): Inflammation and infection of pulp and periapical tissues. Oral. Surg. 34, 486-497.

Schroder, U. (1985): Effects of calcium hydroxide-containing pulp-capping agents on pulp cell migration, proliferation and differentiation. J. Dent. Res. 64 (Special Issue), 541-548.

Sicher, H. and Bhaskar, S. N. (1990): Pulp. In: Orban's Oral histology and Embryology. Mosby, St. Louis. pp. 139-179.

Ten Cate, A. R. (1985): Odontoblasts. J. Dent. Res. 64 (Special Issue), 549-551.

Tonder, K. J. H. and Kvinnsland, I. (1983): Micropuncture measurements of interstitial fluid pressure in normal and inflamed dental pulp in cats. J. Endod. 9, 105-109.

Tonder, K. J. H. and Naess, G. (1979): Microvascular pressure in the dental pulp and gingiva in cats. Acta Odontol. Scand. 37, 161-168.

Tronstad, L. (1974): Reaction of the exposed pulp to dycal treatment. Oral. Surg. 38, 945-953.

Tronstad, L. and Mjor, I. A. (1972): Capping of the inflamed pulp. Oral. Surg. 34, 477-485.

Vrbošek, V. (1981): Bacterial antigens and unspecific dental pulp stimulation (in Slovenian). Thesis, University of Ljubljana.

Wenneberg, A., Mjor, I. A. and Heide, S. (1982): Rate of formation of regular and irregular secondary dentin in monkey teeth. Oral Surg. 54, 232-237.

Yamamura, T. (1985): Differentiation of pulpal cells and inductive influences of various matrices with reference to pulpal wound healing. J. Dent. Res. 64 (Special Issue), 530-540. 